\title{
Citizen-Centered Mobile Health Apps Collecting Individual-Level Spatial Data for Infectious Disease Management: Scoping Review
}

\author{
Felix Nikolaus Wirth ${ }^{1,2}$, MSc; Marco Johns ${ }^{1,2}$, MSc; Thierry Meurers ${ }^{1,2}$, MSc; Fabian Prasser ${ }^{1,2}$, PhD \\ ${ }^{1}$ Berlin Institute of Health, Berlin, Germany \\ ${ }^{2}$ Charité - Universitätsmedizin Berlin, corporate member of Freie Universität Berlin, Humboldt-Universität zu Berlin, and Berlin Institute of Health, \\ Berlin, Germany
}

\section{Corresponding Author:}

Felix Nikolaus Wirth, MSc

Berlin Institute of Health

Charitéplatz 1

Berlin, 10117

Germany

Phone: 4915773326360

Email: felix-nikolaus.wirth@charite.de

\begin{abstract}
Background: The novel coronavirus SARS-CoV-2 rapidly spread around the world, causing the disease COVID-19. To contain the virus, much hope is placed on participatory surveillance using mobile apps, such as automated digital contact tracing, but broad adoption is an important prerequisite for associated interventions to be effective. Data protection aspects are a critical factor for adoption, and privacy risks of solutions developed often need to be balanced against their functionalities. This is reflected by an intensive discussion in the public and the scientific community about privacy-preserving approaches.

Objective: Our aim is to inform the current discussions and to support the development of solutions providing an optimal balance between privacy protection and pandemic control. To this end, we present a systematic analysis of existing literature on citizen-centered surveillance solutions collecting individual-level spatial data. Our main hypothesis is that there are dependencies between the following dimensions: the use cases supported, the technology used to collect spatial data, the specific diseases focused on, and data protection measures implemented.

Methods: We searched PubMed and IEEE Xplore with a search string combining terms from the area of infectious disease management with terms describing spatial surveillance technologies to identify studies published between 2010 and 2020 . After a two-step eligibility assessment process, 27 articles were selected for the final analysis. We collected data on the four dimensions described as well as metadata, which we then analyzed by calculating univariate and bivariate frequency distributions.

Results: We identified four different use cases, which focused on individual surveillance and public health (most common: digital contact tracing). We found that the solutions described were highly specialized, with 89\% (24/27) of the articles covering one use case only. Moreover, we identified eight different technologies used for collecting spatial data (most common: GPS receivers) and five different diseases covered (most common: COVID-19). Finally, we also identified six different data protection measures (most common: pseudonymization). As hypothesized, we identified relationships between the dimensions. We found that for highly infectious diseases such as COVID-19 the most common use case was contact tracing, typically based on Bluetooth technology. For managing vector-borne diseases, use cases require absolute positions, which are typically measured using GPS. Absolute spatial locations are also important for further use cases relevant to the management of other infectious diseases.

Conclusions: We see a large potential for future solutions supporting multiple use cases by combining different technologies (eg, Bluetooth and GPS). For this to be successful, however, adequate privacy-protection measures must be implemented. Technologies currently used in this context can probably not offer enough protection. We, therefore, recommend that future solutions should consider the use of modern privacy-enhancing techniques (eg, from the area of secure multiparty computing and differential privacy).
\end{abstract}

(JMIR Mhealth Uhealth 2020;8(11):e22594) doi: 10.2196/22594 


\section{KEYWORDS}

pandemic; epidemic; infectious disease management; mobile apps; automated digital contact tracing; mobility tracking; outbreak detection; location-based risk assessment; public health; informatics; app; infectious disease; COVID-19; review

\section{Introduction}

\section{Background}

In December 2019, a novel coronavirus (SARS-CoV-2) appeared and rapidly spread around the world. COVID-19, the disease associated with the virus, can cause severe respiratory illness, is highly transmissible among humans [1], and is often difficult to detect due to asymptomatic courses [2]. The outbreak was declared a Public Health Emergency of International Concern and has implications for global health and economic development alike.

To contain the pandemic, nonpharmaceutical interventions have been implemented on a broad scale as a rapid response. They aim at slowing down or interrupting the infection process by breaking the chain of infections [3]. Examples include individual measures such as social distancing, the wearing of masks, and self-isolation [4] as well as public measures including travel restrictions [5], closure of public institutions and businesses [6], quarantines, and curfews [7]. Many of these measures, however, come with drastic socioeconomic consequences [8]. For example, it has been estimated that the reduction in economic activity resulting from the lockdown will have an impact on the Euro area that is three to four times larger than the impact of the global financial crisis of 2007 and 2008 [9]. Further, it is expected that the pandemic and subsequent lockdowns will lead to mental health problems [10] and excess mortality indirectly related to COVID-19 [11]. Hence, there is considerable pressure to move toward policies that will allow a return of economic and social life to the former state while effectively containing the spread of SARS-CoV-2. This can probably best be achieved by replacing large-scale interventions with local or even individual interventions based on testing, tracing, and targeted quarantine [12,13]. This, however, requires effective means of disease surveillance.

For example, contact tracing is traditionally the manual process of identifying past contacts of a person with an infectious disease [14]. However, manual contact tracing is time and labor intensive, which is a challenge considering the scale of the COVID-19 pandemic. Thus, many believe that citizen-centered participatory digital tools are required [15]. Most notably, automated digital contact tracing via mobile apps (henceforth simply called contact tracing) is discussed in public media and scientific literature alike [16-20], and according infrastructures have been implemented in several countries. At the time of writing, the COVID Tracing Tracker Project lists more than 40 different apps [21].

An important aspect of automated contact tracing is its reliance on citizen-centered mobile apps collecting individual-level spatial data. The broad adoption of such apps is considered a major prerequisite for associated interventions to be effective [22], and it has been argued that data security and privacy aspects are a critical factor for adoption [22,23]. This is reflected by an intense discussion in the public and the scientific community about privacy-preserving solutions to the contact tracing problem [24,25] and the multitude of established solutions and projects [26-30]. However, often there is a trade-off between privacy risks associated with and the functionalities provided by solutions developed. The German contact tracing app, for example, focuses solely on contacts and builds upon an infrastructure with few central services [31]. As a result, it only supports contact tracing and does not offer any additional functionalities for disease surveillance.

There are, however, several additional use cases that can be implemented with location-based mobile health apps that could help with managing the current and future pandemics. A few unsystematic overviews of technologies currently implemented to manage the COVID-19 pandemic have been published $[23,32,33]$. For example, Boules and Geraghty [16] have listed several projects: HealthMap, a system that aggregates and maps informal information sources such as online social networks to create risk maps; WorldPop and EpiRis, which use location-based services and other sources of data to model human mobility to predict the spread of COVID-19; and the close contact detector, a solution that uses data on the movement of people to identify individuals with close contact to people who are infected. Other authors suggested the use of digital thermometers, smart watches, or other mobile health devices to remotely monitor vital signs and potential symptoms of at-risk individuals [32,33]. In addition, there are reviews focusing on solutions for health care professionals [34,35]. What is lacking, however, is a structured analysis of important aspects relevant to the technical properties and functionalities of current and future citizen-centered solutions.

\section{Objectives}

The objectives of this work are to inform the current discussions and to support the development of participatory disease surveillance technologies, providing an optimal balance between privacy protection and pandemic control. In our opinion, these objectives are best met by a systematic analysis of existing literature. Our main hypothesis is that there are dependencies between the specific diseases focused on, use cases supported, technology used, and data protection measures implemented. Furthermore, we hypothesize that these dependencies influence the possible design space of solutions. In particular, we focus on the following dimensions:

- Use case dimension: Many of the current discussions are focused on technical aspects and their consequences in terms of privacy risks. When considering use cases, the discussion seems to be focused only on contact tracing. Hence, we aim to obtain a broader view of the existing solutions' functionalities to facilitate the evaluation of novel concepts and to guide the development of future solutions.

- Technology dimension: Many recent developments are based on Bluetooth Low Energy (BLE), which is a relatively new technology, and GPS receivers. However, the choice of technology can also impact the functionalities that can 
be implemented. To potentially broaden the design space for future tools, our objective is to get a broad overview of the technologies that have been used in prior projects and to analyze potential relationships to the use cases that have been implemented.

- Disease dimension: There are specific properties of SARS-CoV-2 that have contributed to the characteristic nature of the current pandemic. To determine whether existing solutions can serve as a blueprint for future work, we aim to investigate use cases and technologies in relationship to disease properties such as the path of transmission, infectiousness, and disease-associated symptoms.

- Data protection dimension: Finally, privacy protection is seen as an essential factor in current discussions on apps related to COVID-19. To derive a complete picture of data protection measures that can be implemented in this context, we aim to compile an overview of measures implemented in prior projects.

\section{Methods}

We performed a scoping review, as this type of review is best suited to map research activities in a broad and heterogeneous field [36]. Where applicable, we followed the guidelines of the PRISMA-ScR (Preferred Reporting Items for Systematic Reviews and Meta-Analyses extension for scoping reviews) [37]. No review protocol was registered for this study. From the objectives, we derived the following inclusion criteria for articles:

- Infectious disease management: Solutions described must be focused on managing epidemics or pandemics of a pathogen, which is communicable directly from human-to-human, such as COVID-19, or transmitted by a vector, such as Dengue.

- Mobile health apps: Solutions proposed must be centered around a mobile device or app, which is used for data collection. Articles may describe a concept or an actual implementation. For instance, articles describing methods for modeling population-level disease spread but not covering methods of data collection were not included.

- Based on individual-level spatial data: Solutions described must be enabled by collecting individual-level spatial data (individual persons or small groups such as families), either absolute (eg, location determined via GPS) or relative (eg, contacts determined using Bluetooth), either dynamically (eg, regular updates to track movements) or statically (eg, manual entry of living address).
- Citizen-centered solution: Data must be collected either automatically or manually by regular citizens using their own devices. In contrast, solutions focused on data collection by professionals within a health care or surveillance context (such as apps for professional contact tracers) were excluded.

An article needed to fulfill all these criteria to be included. Furthermore, all papers were required to be written in English, published between the years 2010 and 2020 (final search performed on June 19, 2020), and scientific peer-reviewed papers containing original work.

We searched both PubMed and IEEE Xplore, as the topic of this review is placed at the intersection of medicine and technology. First, we specified a set of keywords describing the general context. At least one of these keywords needed to be contained in the title of articles checked for eligibility: epidemic, pandemic, contact tracing, proximity tracing, surveillance, or infectious. Second, we specified a set of keywords covering the complete spectrum of relevant technology. At least one of these keywords needed to be contained in either the title or the abstract of the articles: mobile, wearables, smartphone, app, cellular network, NFC (near-field communication), barcode, QR (Quick Response) code, GPS, Wi-Fi, Bluetooth, RFID (radio-frequency identification), or magnetometer. Search results were exported as comma-separated values files, harmonized, and imported into a consolidated spreadsheet. In total, we found 1133 articles, of which 646 were identified via PubMed and 487 via IEEE Xplore.

Figure 1 shows an overview of the selection process. The articles were selected in two steps. In each step, articles were randomly assigned to two authors for assessment, and cases of disagreement were discussed among all authors for consent. In a first screening step, the title and abstract were evaluated regarding the eligibility criteria. Articles were kept if the title or abstract did not provide for a decision on exclusion or inclusion. In total, 99 articles were included after this screening step. Next, the full texts of the selected articles were compared against the eligibility criteria. After this analysis, 27 articles were regarded as finally relevant and were included into the data extraction and analysis process. In this process, we extracted the year and free text for the remaining variables and mutually created categories from the text extracted. The data is available in Multimedia Appendix 1.

Table 1 illustrates the data items collected from the final selection of articles to answer the research questions previously outlined. 
Figure 1. Overview of the selection process.
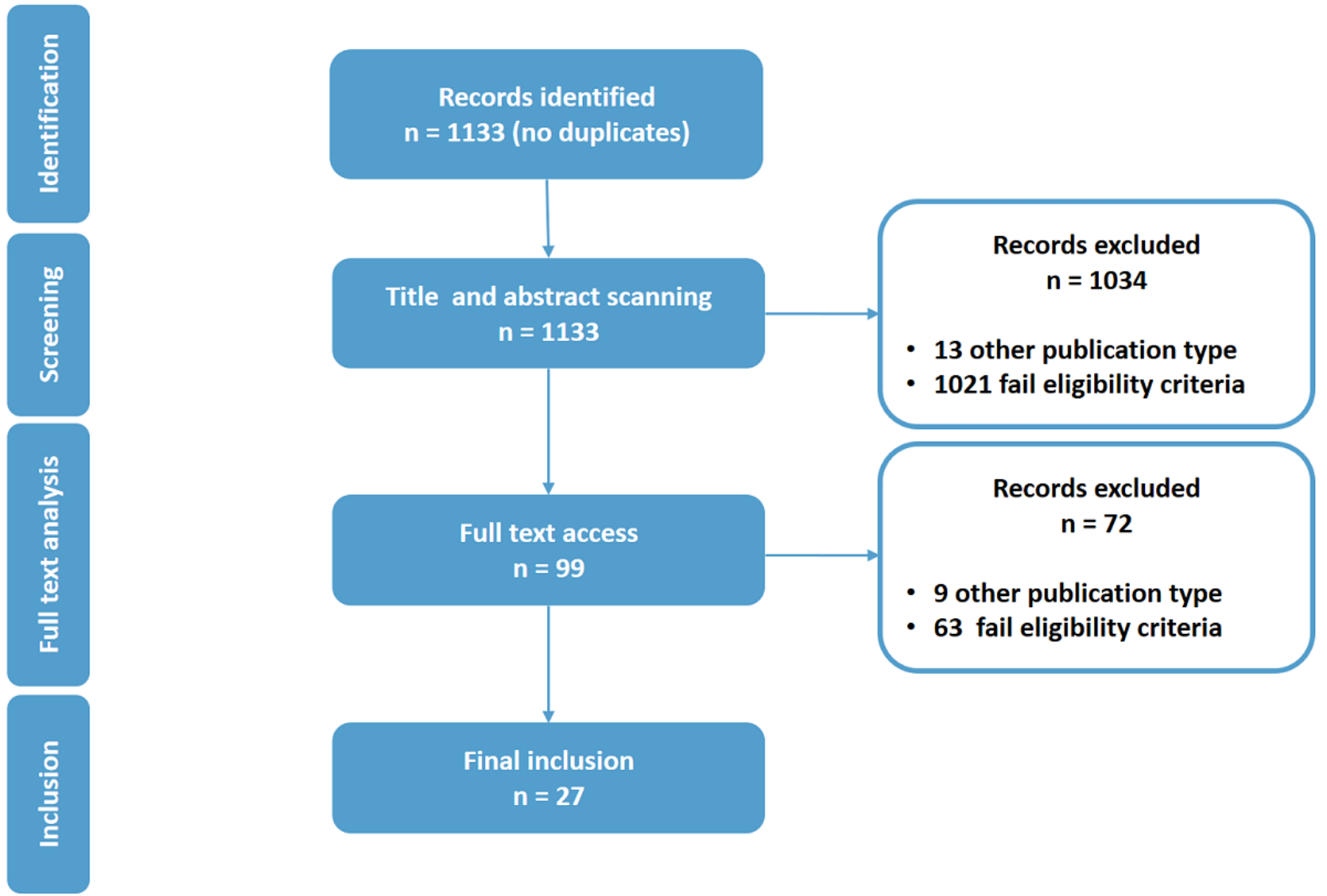

Table 1. Data items collected.

\begin{tabular}{lll}
\hline Variable & Examples & Definition \\
\hline Year & 2010, 2018,... & The year the article describing the solution was published \\
$\begin{array}{l}\text { Use cases } \\
\text { Sensor technolo- } \\
\text { gies }\end{array}$ & Contact tracing, outbreak detection,,.. & The infectious disease management processes addressed by the solution \\
$\begin{array}{l}\text { Disease } \\
\text { Data protection }\end{array}$ & COVID-19, influenza-like illness,... & The technology used for spatial tracking \\
\hline
\end{tabular}

The data collected corresponds with the dimensions investigated. First, we collected the year of publication to analyze developments over time. Second, in accordance with the first dimension, data was collected on the use case or cases supported by the apps. Third, in accordance with the second dimension, the sensor technology used to obtain spatial data was recorded. Fourth, in accordance with the third dimension, we collected data on the diseases that the solutions aimed to manage. Finally, in accordance with the fourth dimension, we recorded the data protection mechanisms used.
During data collection, the spreadsheet document was extended to work as a chartering form containing all data items. In accordance with our research questions, data was then analyzed by calculating frequency distributions of variables' values and by analyzing the relationships between variables using heat maps, visualizing the combined frequencies of their values.

\section{Results}

\section{Overview}

Table 2 shows the final selection of articles together with the collected data. 
Table 2. Selected articles and data collected.

\begin{tabular}{|c|c|c|c|c|c|}
\hline Author & Year & Use case & Disease & Sensor technology & Data protection measures \\
\hline Abbas and Michael [38] & 2020 & Contact tracing & COVID-19 & Bluetooth & $\begin{array}{l}\text { Data minimization, } \\
\text { pseudonymization }\end{array}$ \\
\hline Abeler et al [24] & 2020 & Contact tracing & COVID-19 & Bluetooth & Pseudonymization \\
\hline Ackley et al [39] & 2020 & Outbreak detection & $\mathrm{ILI}^{\mathrm{a}}$ & GPS, $\mathrm{IP}^{\mathrm{b}}$ address geolocation & $\begin{array}{l}\text { Geospatial aggregation, } \\
\text { pseudonymization, temporal aggre- } \\
\text { gation }\end{array}$ \\
\hline Barrat et al [40] & 2014 & Contact tracing & Generic & Bluetooth & None \\
\hline Chan et al [41] & 2020 & Outbreak detection & COVID-19 & Manual entry & $\begin{array}{l}\text { Consent, data minimization, } \\
\text { pseudonymization }\end{array}$ \\
\hline Farrahi et al [42] & 2014 & Contact tracing & Generic & Bluetooth, phone logs & Consent, temporal aggregation \\
\hline Ferretti et al [43] & 2020 & Contact tracing & COVID-19 & Code scanning, GPS & Consent, transparency \\
\hline Jeong et al [44] & 2019 & Contact tracing & Generic & Magnetometer & Geospatial aggregation \\
\hline Kim et al [45] & 2019 & Outbreak detection & ILI & Not specified & None \\
\hline Kim et al [46] & 2016 & $\begin{array}{l}\text { Location-based risk assess- } \\
\text { ment, mobility tracking }\end{array}$ & Zika & GPS & None \\
\hline Leal Neto et al [47] & 2017 & Outbreak detection & Generic & GPS, manual entry & Data minimization \\
\hline Leal Neto et al [48] & 2020 & Outbreak detection & Generic & Not specified & Consent \\
\hline Lwin et al [49] & 2014 & Outbreak detection & Dengue & GPS, manual entry & None \\
\hline Michael and Abbas [50] & 2020 & Contact tracing & COVID-19 & Bluetooth & Pseudonymization \\
\hline Miller et al [51] & 2018 & $\begin{array}{l}\text { Location-based risk assess- } \\
\text { ment, outbreak detection }\end{array}$ & ILI & GPS & None \\
\hline Navin et al [52] & 2017 & $\begin{array}{l}\text { Location-based risk assess- } \\
\text { ment, outbreak detection }\end{array}$ & Generic & Not specified & None \\
\hline Nguyen et al [53] & 2017 & Contact tracing & Generic & Magnetometer & Geospatial aggregation \\
\hline Olson et al [54] & 2017 & Outbreak detection & Gastroenteritis & Manual entry & None \\
\hline Okumura [55] & 2019 & Contact tracing & Generic & GPS, GSM ${ }^{\mathrm{c}}$ & Data minimization \\
\hline Prieto et al [56] & 2015 & Outbreak detection & ILI & Manual entry & None \\
\hline Rodriguez-Valero et al [57] & 2018 & Outbreak detection & Zika & GPS & Pseudonymization \\
\hline Sugiura et al [58] & 2010 & Outbreak detection & Generic & Manual entry & None \\
\hline Tripathy et al [59] & 2020 & Contact tracing & COVID-19 & Bluetooth & None \\
\hline Vazquez-Prokopec et al [60] & 2013 & Mobility tracking & Generic & GPS & None \\
\hline Wang et al [61] & 2020 & Contact tracing & COVID-19 & GPS & None \\
\hline Yasaka et al [20] & 2020 & Contact tracing & COVID-19 & Code scanning & Pseudonymization \\
\hline Zhang et al [62] & 2013 & Contact tracing & Generic & Bluetooth & None \\
\hline
\end{tabular}

${ }^{a}$ ILI: influenza-like illness.

bIP: Internet Protocol.

${ }^{\mathrm{c}}$ GSM: Global System for Mobile Communications.

As can be seen, more than half of the articles have been published in the last 3 years, indicating a current interest in the topic. In the following sections, we will present further analyses to investigate the questions outlined in the "Objectives" section.

\section{Use Case Dimension}

The first objective of this study was to take a broader look at functionalities of mobile health apps using spatial data for infectious disease management.
Within the selected set of articles, we identified four use cases, which we assigned to two distinct categories. The first category is user-centered services (ie, solutions focusing on individual health). The first such use case is automated contact tracing, where data on contacts between individuals and on infections is used to determine exposure risk and to notify individuals if necessary. The second such use case is location-based risk assessment, where the locations of individuals are used to warn them when entering areas with high disease activity. The latter 
type of solutions might also be used to estimate individual exposure risk.

The second category is disease surveillance (ie, solutions focusing on population health), which involves collecting and analyzing data to monitor the occurrence of diseases within the population with the aim to support public health interventions. The first use cases in this category were outbreak detection, which can include syndromic surveillance (eg, based on symptoms reported or data about confirmed cases to determine areas with disease activity). The second such use case is mobility tracking, where patterns about the movement of larger groups of individuals are determined to support various analyses, such as the modeling of disease dynamics.

We emphasize that, although these use cases can be implemented independently of one another, there are obvious relationships. For example, data collected via an outbreak detection app could also be used to provide location-based risk assessment services. Moreover, both individual-level as well as population-level use cases ultimately aim to protect the entire population and its individuals. Table 3 illustrates how often the use cases have been described.

Table 3. Overview of use cases covered.

\begin{tabular}{ll}
\hline Use case & Articles, $\mathrm{n}$ \\
\hline Contact tracing & 13 \\
Outbreak detection & 12 \\
Location-based risk assessment & 3 \\
Mobility tracking & 2 \\
\hline
\end{tabular}

Within the 27 papers, automated contact tracing has been addressed $13(48.1 \%)$ times. Outbreak detection was covered by $12(44.4 \%)$ of the papers. Location-based risk assessment was described in $3(11.1 \%)$ papers, and mobility tracking in 2 (7.4\%) papers.

As mentioned before, different use cases can also be combined with each other. However, the articles identified are quite specialized: 24 (89\%) focused on a single use case. The remaining $3(11 \%)$ all combined location-based risk assessment with an additional use case.

Current discussions on participatory mobile health solutions in the context of COVID-19 largely focus on automated contact tracing only. To gain insights into potential directions for future developments, we also analyzed which use cases have been described for which disease (see "Disease Dimension" section for more details). Figure 2 shows the common distribution of use cases described and diseases focused on.

Figure 2. Relationships between diseases and use cases described. ILI: Influenza-like illness.

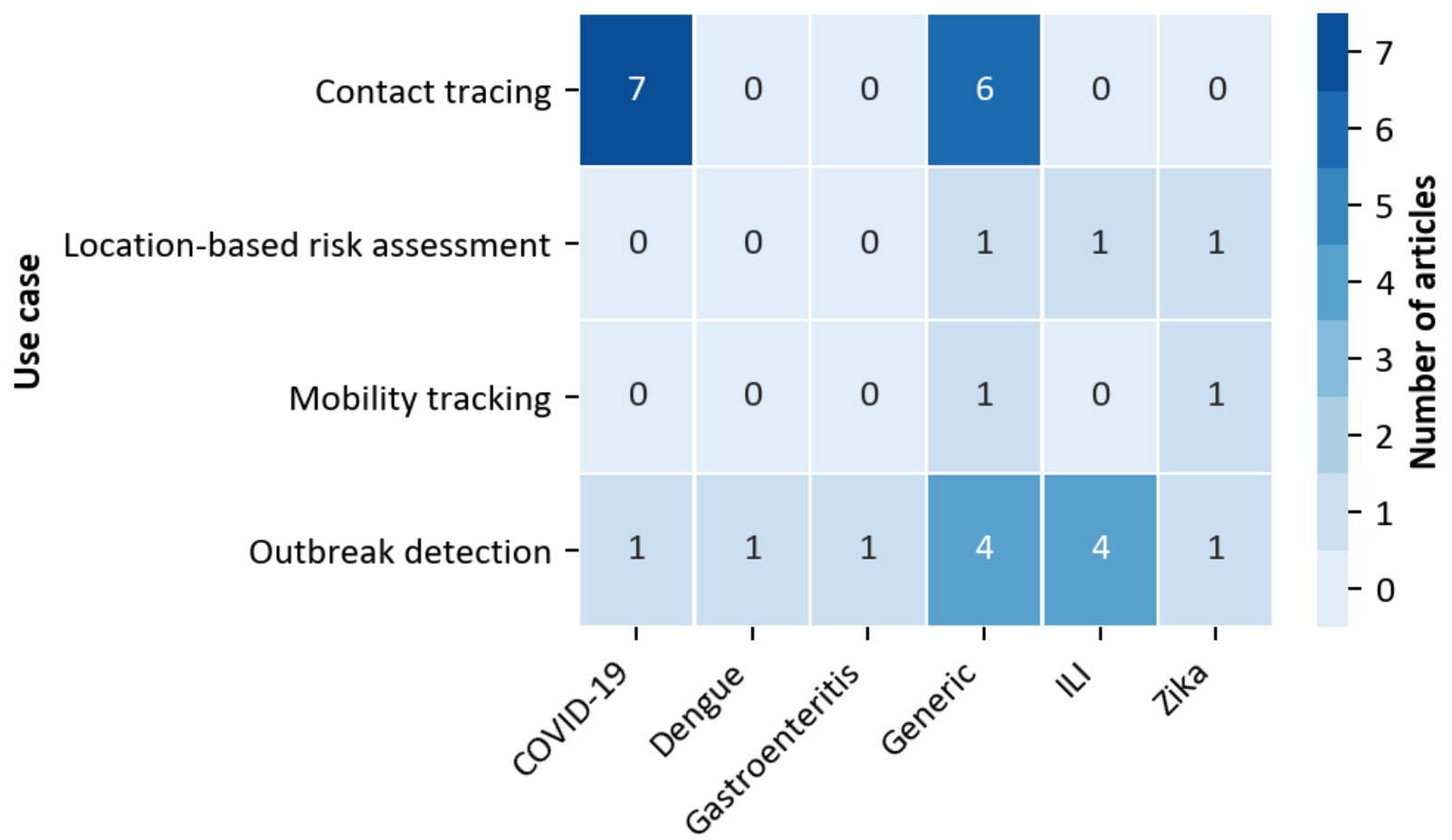

Disease 
As can be seen, in accordance with current discussions, papers that address COVID-19 focused nearly exclusively on automated contact tracing. For other diseases, however, several further use cases have been described. Moreover, a wide range of generic concepts has been proposed independently of a concrete disease. These focused mainly on automated contact tracing and outbreak detection. We also analyzed in which year solutions for certain use cases have been described in the articles analyzed. An interesting finding is that $69 \%(9 / 13)$ of the papers focusing on automated contact tracing were published in the years 2019 and 2020 , while $71 \%(10 / 14)$ of all papers focusing on other use cases were published in 2018 or earlier.

\section{Technology Dimension}

In this dimension, we aimed to obtain an overview of technologies that have been proposed to implement the solutions identified. Moreover, we wanted to gather insights into the degree to which technical implementation options and supported functionalities are related to one another.
In total, we identified eight distinct technologies that have been proposed for collecting spatial data: (1) GPS receivers collect absolute coordinates using a satellite system, (2) Bluetooth can be used to estimate the physical proximity between two devices and hence collect relative spatial data (ie, contacts between people), (3) manual entry refers to the manual recording of spatial information such as a living address, (4) magnetometers can be used to measure the small magnetic interferences produced in proximity to a second magnetometer, (5) code scanning refers to the scanning of a code such as a $\mathrm{QR}$ code to record a contact or a certain location, (6) Global System for Mobile Communications (GSM) can be used to estimate the absolute geospatial position, (7) Internet Protocol (IP)-based geolocation refers to the estimation of the absolute location by checking the range a user's IP address is assigned to, and (8) phone $\operatorname{logs}$ refer to the relative position between people by analyzing phone calling lists and text messages. The frequency with which individual technologies were used is displayed in Table 4.

Table 4. Overview of technologies used for collecting spatial data (three solutions with unspecified technology excluded).

\begin{tabular}{lc}
\hline Sensor technology & Articles, n \\
\hline GPS & 10 \\
Bluetooth & 7 \\
Manual entry & 6 \\
Code scanning & 2 \\
Magnetometer & 2 \\
Phone logs & 1 \\
IP address geolocation & 1 \\
GSM $^{\text {b }}$ & 1 \\
\hline
\end{tabular}

${ }^{\mathrm{a}} \mathrm{IP}$ : Internet Protocol.

${ }^{\mathrm{b}}$ GSM: Global System for Mobile Communications.

Out of 27 articles, GPS, as the most frequent technology, has been described $10(30.3 \%)$ times, followed by Bluetooth, which was mentioned $7(21.1 \%)$ times, and manual entry, which was described in $6(18.2 \%)$ papers. Magnetometers and code scanning were each mentioned in $2(6.1 \%)$ papers. GSM, IP-based geolocation, and phone logs were each suggested in $1(3.0 \%)$ paper. It is noteworthy that 3 articles did not explicitly specify the technology suggested for collecting spatial data (see $[45,48,52])$. Moreover, it can be noted, that none of the papers described solutions using wireless local area network access points. We also analyzed the ability of the solutions described to track an absolute position (longitude and latitude) or a relative position (proximity to other devices): $60 \%(\mathrm{n}=18)$ are able to measure absolute positions, while 40\% ( $n=12)$ can only determine relative positions.

When looking at the time at which papers have been published that use the two most common technologies, GPS and Bluetooth, spikes in the frequency of mentions of Bluetooth can be seen in the years 2013-2014 (the time when the technology first became available on a large scale on commodity devices) and 2020 (the time of the SARS-CoV-2 pandemic). Articles about solutions using GPS have been published continuously over time.

To study how technology might influence the use cases implemented, we analyzed the relationships between both aspects. The result is shown in Figure 3. 
Figure 3. Relationship between use cases and technology used to capture spatial data (three solutions with unspecified technology excluded). GSM: Global System for Mobile Communications; IP: Internet Protocol.

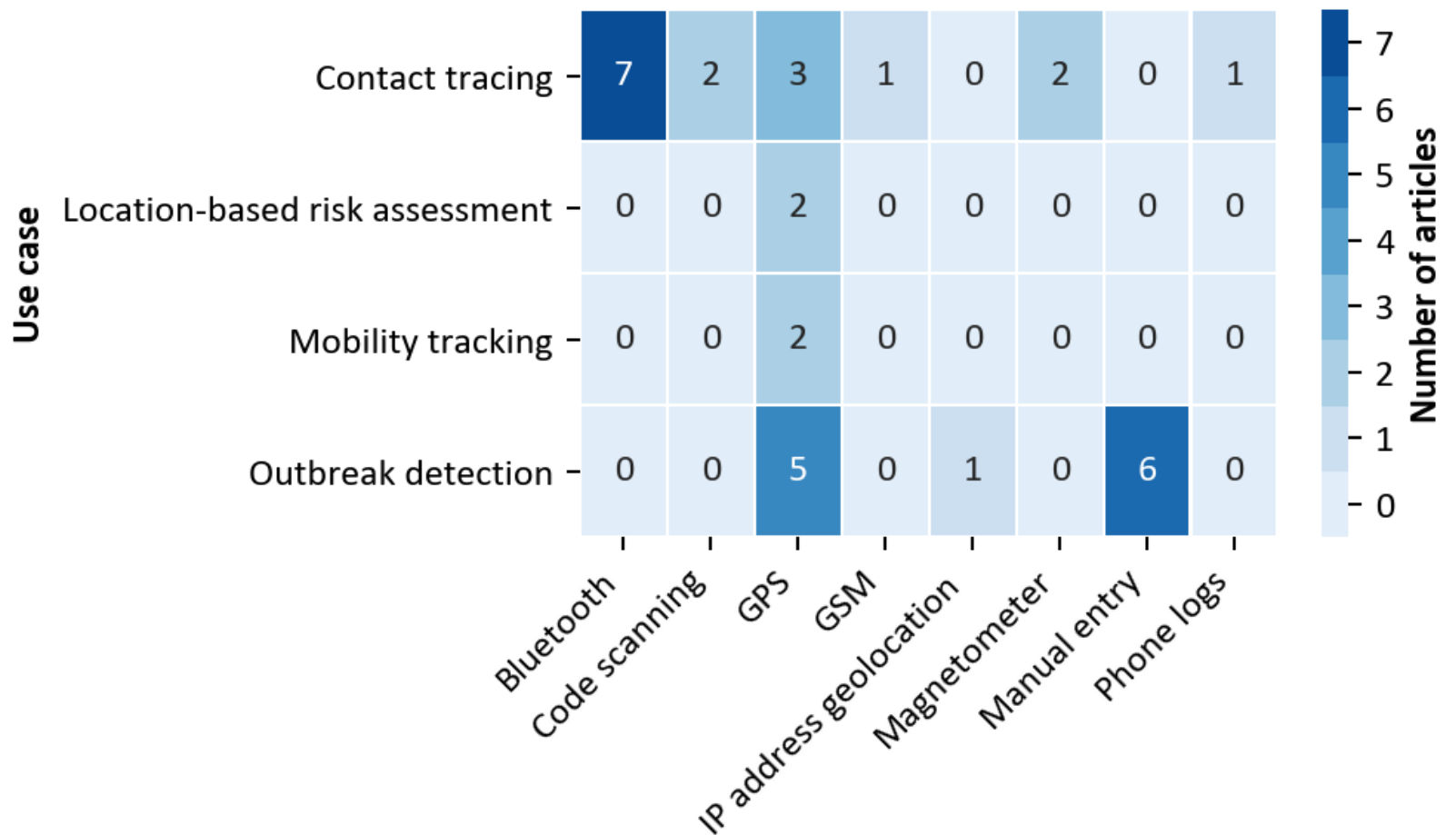

\section{Sensor technology}

It can be seen that Bluetooth is a common technology that has only been used to implement contact tracing (7 papers). Manual data entry is also common but has only been used for outbreak detection (6 papers). GPS, however, is a frequent and versatile technology that has been proposed or implemented for all four use cases identified (12 papers in total). The two remaining technologies that have been suggested more than once are code scanning and magnetometers. Both have only been used to support contact tracing solutions.

\section{Disease Dimension}

An important objective of our analysis was to study potential relationships between disease properties and use cases as well as technologies used. We identified five specific diseases addressed: (1) COVID-19 and (2) influenza-like illness (ILI) are mainly transmitted by droplet infection and cause symptoms like coughing and fever; (3) Dengue and (4) Zika are transmitted mainly by mosquitos and cause symptoms like rash, vomiting, and fever; and (5) gastroenteritis is mainly transmitted by smear infection and causes symptoms like diarrhea, vomiting, and fever. As a first step, we analyzed the frequency with which certain diseases have been addressed by publications. The results are shown in Table 5.

Table 5. Overview of diseases targeted by the solutions analyzed.

\begin{tabular}{ll}
\hline Disease & Articles, $\mathrm{n}$ \\
\hline Generic & 11 \\
COVID-19 & 8 \\
ILI $^{\mathrm{a}}$ & 4 \\
Zika & 2 \\
Dengue & 1 \\
Gastroenteritis & 1 \\
\hline
\end{tabular}

${ }^{\mathrm{a}}$ ILI: influenza-like illness.

Out of 27 articles, $11(40.7 \%)$ of the papers described generic solutions not designed for a specific disease. Of the remaining papers, 8 (29.6\%) mentioned COVID-19, 4 (14.8\%) mentioned
ILI, 2 (7.4\%) mentioned Zika, 1 (3.7\%) mentioned Dengue, and $1(3.7 \%)$ mentioned gastroenteritis.

We then analyzed whether there was a specific relationship between disease properties and the use cases suggested. For this 
purpose, we again refer to Figure 3. It can be seen that, obviously, automated contact tracing has only been suggested for highly infectious human-to-human transmissible diseases. For other diseases that are transmitted by vectors, outbreak detection, mobility tracking, and location-based risk assessment are more common. To study whether a similar relationship can also be found regarding the sensor technology, we compiled the data presented in Figure 4.

Figure 4. Relationship between diseases and sensor technology (three solutions with unspecified technology excluded). ILI: Influenza-like illness; GSM: Global System for Mobile Communications; IP: Internet Protocol.

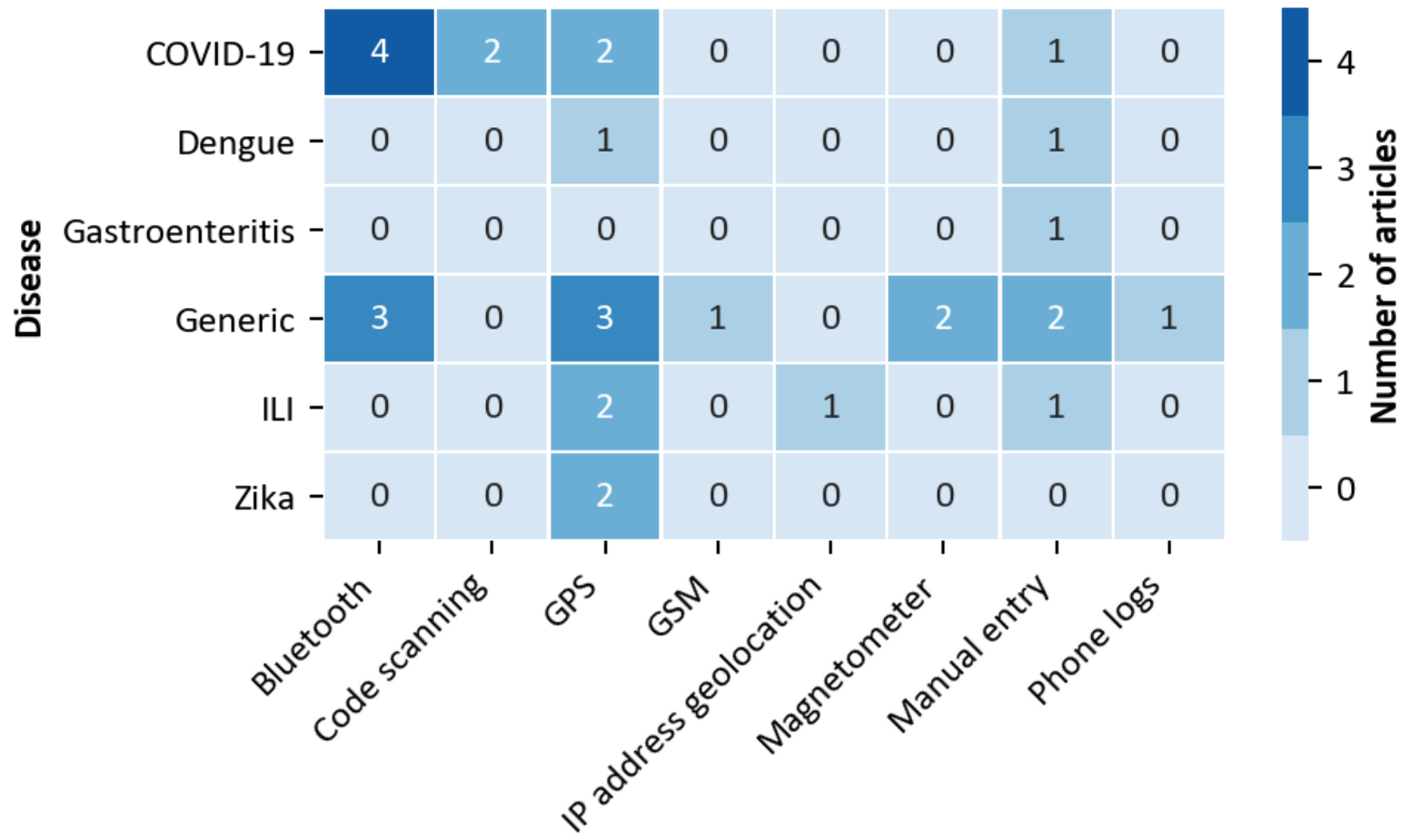

Sensor technology

Figure 4 shows that Bluetooth, which is a solution for exclusively tracking relative spatial data (ie, contacts), has only been suggested for generic disease tracking or for managing COVID-19. In contrast, technologies for tracking absolute positions (primarily GPS) have more frequently been proposed for other diseases. It is also worth mentioning that 2 papers suggested the use of code scanning for COVID-19, which is a versatile technology that can be used to track both absolute and relative spatial data.

\section{Data Protection Dimension}

Data protection is a central aspect in many discussions on automated contact tracing and related use cases. To gain insights into measures that have been proposed, we first analyzed the protection measures mentioned in the selected articles.

We identified six different data protection measures: (1) pseudonymization, which refers to the replacement of identifying information with random meaningless identifiers;
(2) geospatial and (3) temporal aggregation, which refers to techniques for reducing the uniqueness of data; (4) data minimization, which is a privacy-by-design measure implying that as little information as necessary is stored and processed; (5) consent, which means that users are explicitly asked to permit data processing, typically within a study setting; and (6) transparency, which refers to the general principle of communicating which data is stored and how it is processed.

Table 2 shows that $52 \%(n=14)$ of the papers did list privacy protection measures, while $48 \%(n=13)$ of papers did not mention data protection aspects. When taking a look at the time in which the individual papers were published, it can be seen that there is a trend toward more consideration of data protection aspects in recent years. In total, data protection measures were only mentioned in one of the papers published before 2017.

We then analyzed how often the individual measures were suggested or implemented. The results are shown in Table 6. 
Table 6. Data protection measures mentioned in the articles selected.

\begin{tabular}{ll}
\hline Data protection measure & Articles, $\mathrm{n}$ \\
\hline Pseudonymization & 7 \\
Consent & 4 \\
Data minimization & 4 \\
Geospatial aggregation & 3 \\
Temporal aggregation & 2 \\
Transparency & 1 \\
\hline
\end{tabular}

Table 6 shows that pseudonymization, out of 27 articles, has been addressed 7 (33.3\%) times, followed by consent and data minimization, which were each mentioned 4 (19.0\%) times. Moreover, geospatial aggregation was described 3 (14.2\%) times, temporal aggregation $2(9.5 \%)$ times, and transparency $1(4.7 \%)$ time. These measures can be categorized into organizational and technical measures. The organizational measures transparency and consent have been mentioned 5 $(23.8 \%)$ times, and the remaining technical measures have been mentioned $16(76.2 \%)$ times.
Regarding the relationships between use cases and data protection measures, it must be noted that all 4 use cases identified were potentially privacy-invading. However, data protection measures have not been mentioned in any of the papers covering location-based risk assessment or mobility tracking.

Finally, we also studied the relationship between technologies and protection measures proposed. The results are shown in Figure 5.

Figure 5. Sensor technologies utilized and protection methods mentioned (three solutions with unspecified technology excluded) GSM: Global System for Mobile Communications; IP: Internet Protocol.

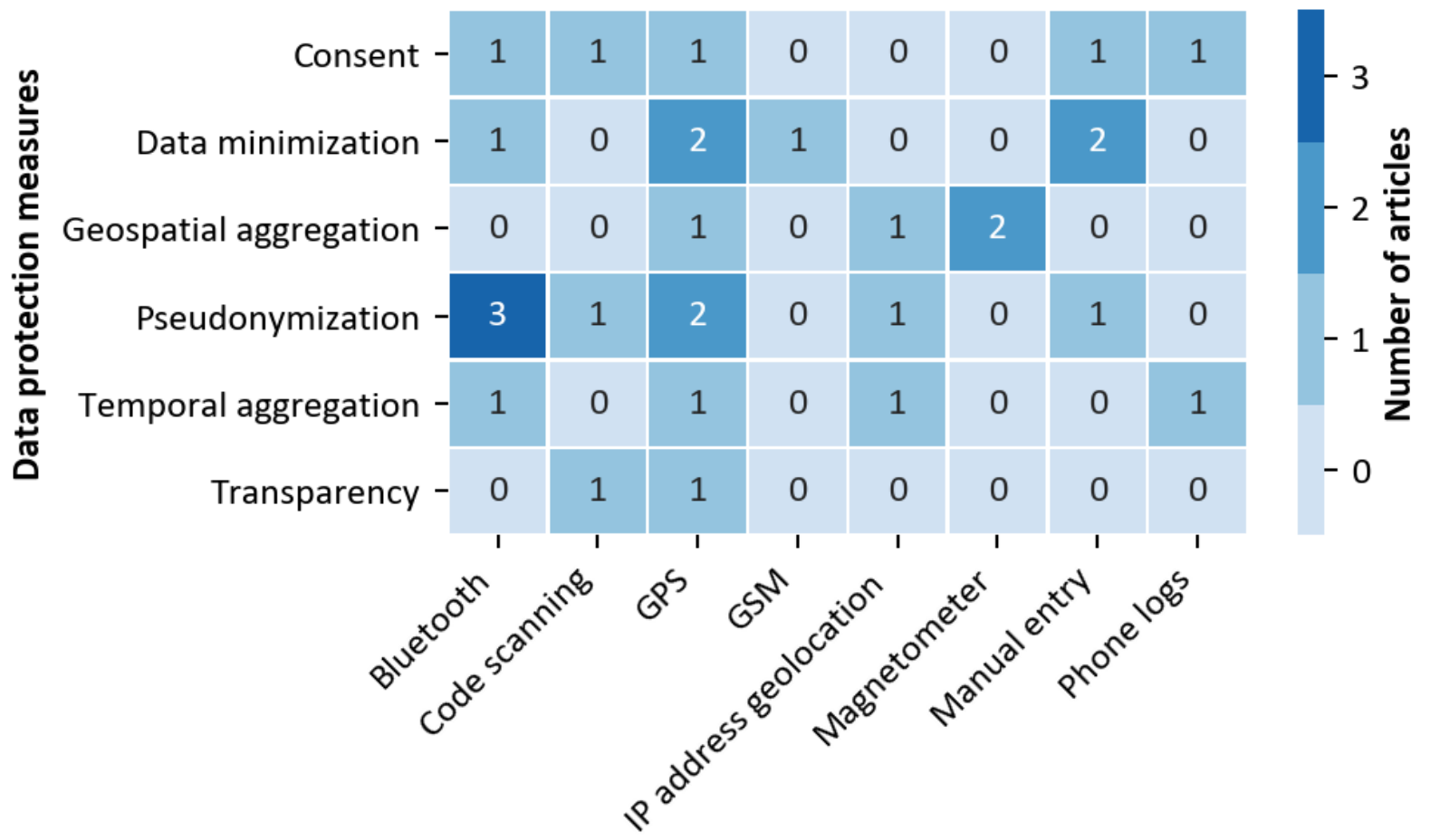

\section{Sensor technology}

It can be seen that many different protection measures have been mentioned in papers focusing on the popular GPS sensor technology. Several measures have also been suggested for protecting Bluetooth-based solutions including pseudonymization, which is particularly important in this context and implemented by exchanging random, pseudonymous identifiers. Most other mentions of protection measures are used in papers focusing on various types of sensors for absolute spatial data other than GPS.

\section{Discussion}

\section{Use Case Dimension}

To broaden the discussion on technical solutions to combat COVID-19 and to propose extension to their functionalities, we first focused on identifying specific use cases. Although most recent publications focus on automated contact tracing, we have identified three additional use cases. Use cases can be grouped 
into two categories: user-centered services and disease surveillance. All these functionalities have already been discussed and used in the context of COVID-19 $[15,16]$ but in an isolated manner. Future work could, therefore, focus on developing concepts and solutions that support different use cases in a combined manner. Our data also indicates, however, that this might be challenging: $89 \%$ (24/27) of all papers analyzed focus exclusively on one use case. Among the notable exceptions is a solution combining outbreak detection with contact tracing for COVID-19 [63].

There might be additional aspects to consider. First, different use cases might be particularly important for different infectious diseases, and the pandemic scale of COVID-19 might be an important factor. We investigated this further in the disease dimension. Second, there might also be technical challenges associated with supporting different use cases and protecting privacy in this process. We investigated this in the technology and data protection dimensions.

\section{Technology Dimension}

We found eight different technologies in the selected literature from which GPS and Bluetooth were most frequently suggested. This is consistent with current discussions on contact tracing apps, which seem to be dominated by BLE. Most technologies were only proposed within the context of a specific use case due to their differences in supporting the measurement of spatial data. For example, Bluetooth has only been proposed for contact tracing, as this technology is only suited for detecting the proximity between individual devices. Moreover, manual data entry has only been suggested for outbreak detection, as it is only suited for tracking static locations such as living addresses. Our results also show that GPS is a highly versatile technology, which has been proposed as a basis for all use cases considered in at least 1 paper. This makes sense, as GPS can be used to collect dynamically absolute locations, which can also be used to determine (to some degree) relative and static spatial data. However, a major challenge arising from this is privacy, which we cover in the data protection dimension.

Our results also suggest that it might be worthwhile to combine different technologies to implement multiple use cases. For example, Bluetooth could be used to implement automated contact tracing, combined with GPS receivers to support location-based risk assessment and mobility tracking. Moreover, this could be combined with manual entry of symptoms or integrated with wearables to collect further data on disease spread. An important prerequisite might be, however, that adequate privacy-enhancing technologies are implemented.

\section{Disease Dimension}

Our results showed that COVID-19 is already the most frequently covered disease in papers on automated contact tracing or related use cases for managing infectious diseases. Obviously, this is due to the scale and global consequences of the COVID-19 pandemic. Moreover, our data shows that most solutions for managing COVID-19 have suggested Bluetooth as the main technology for collecting spatial data. Apart from privacy considerations, this is also well justified by disease properties: COVID-19 is highly infectious, droplet infection is an important route, and there can be a long period of infectiousness before symptom onset. Thus, a technology is needed that can detect close proximity between people at scale with relatively high accuracy [64]. With diseases like Zika and Dengue that are vector-borne, technologies are needed that can capture absolute spatial data (eg, to identify clusters of infections). Code scanning is a technology that can measure absolute and relative spatial data and may, therefore, also be suited for managing diseases like COVID-19. However, code scanning is cumbersome to use, as it is nonautomated and may, therefore, not be well received by the public. It was noticeable that we did not find any articles on several common infectious diseases such as malaria or HIV. The reason is, as we demonstrated, that disease properties do have an influence on the adequacy of solutions to manage them, and solutions focused on these diseases fall out of the scope of this study. One example are apps to estimate the size of local mosquito populations, which are important for managing malaria but typically use special equipment such as mosquito traps [65].

\section{Data Protection Dimension}

Our results show that various types of organizational and technical safeguards have been suggested for protecting privacy when implementing citizen-centered disease surveillance apps. Moreover, more emphasis has been put on this aspect in recent years. Due to the scale of the current pandemic, the topic has become even more important.

There are two use cases that we identified (ie, location-based risk assessment and mobility tracking) for which no protection measures have been proposed in the literature investigated. This is surprising, as both can be considered potentially privacy-invading. Future work will be needed to integrate such use cases into current participatory surveillance apps in a privacy-conscious manner.

Regarding relationships between privacy protection measures and technologies used, we found that more measures have been suggested for technologies that are widely employed and whose sensors provide absolute positions. Our data shows that pseudonymization is an important measure for Bluetooth-based solutions and, thus, especially for the contact tracing use case.

Finally, we would like to add that several techniques have only been mentioned in papers describing concepts and might have been suggested with a rather naïve view on the topic. Most importantly, it is well-known that geospatial as well as temporal aggregation are challenging to implement in a manner offering a high degree of protection [66]. Moreover, even if such an implementation can be developed, it might have significant impacts on the precision of location data [67]. At the same time, solutions focusing on relative spatial data combined with pseudonymization and implemented without continuous data exchange with central services such as the German Corona-Warn-App [31] are considered the current "gold standard" in privacy-preserving automated contact tracing. Future solutions combining relative with absolute spatial data to support further use cases might be built using alternative approaches such as secure multiparty computing protocols [68] and differential privacy [69]. Finally, it is worth mentioning that data protection is not only a technical but also a social 
challenge, as it is highly connected with public reception and trust.

\section{Conclusion}

In this paper, we have analyzed the literature and discovered several relationships between disease properties, use cases, and technologies. To our knowledge, many of these general dependencies have not been described previously. We, therefore, believe that our results can help with enhancing current solutions for contact tracing and related use cases, and with developing novel, more comprehensive concepts. In addition, the described dependencies could support bottom-up development processes leading to solutions that are more likely to stand the test in real-world implementations. Moreover, we have studied data protection measures that have been suggested and discussed their suitability for different technical environments and use cases. We believe that it will be necessary to employ innovative privacy-enhancing technologies to build comprehensive solutions offering additional functionalities such as population surveillance or individual alerting while maintaining the privacy of citizens.

In the future, when more data on the implementation of solutions in the context of the COVID-19 pandemic will become available, we plan to investigate relationships between their properties along the axes considered in this study and outcomes achieved within specific patient populations.

\section{Authors' Contributions}

FP und FW initiated and conceptualized the work. All authors collected and analyzed the data. FP and FW drafted the manuscript. MJ and TM revised the manuscript. All authors read and approved the final manuscript

\section{Conflicts of Interest}

None declared.

\section{Multimedia Appendix 1}

Selection process and collected data.

[XLSX File (Microsoft Excel File), 194 KB-Multimedia Appendix 1]

\section{References}

1. Report of the WHO-China Joint Mission on coronavirus disease 2019 (COVID-19). World Health Organization. 2020. URL: https://www.who.int/docs/default-source/coronaviruse/who-china-joint-mission-on-covid-19-final-report.pdf

2. Lavezzo E, Franchin E, Ciavarella C, Cuomo-Dannenburg G, Barzon L, Del Vecchio C, Imperial College London COVID-19 Response Team, et al. Suppression of COVID-19 outbreak in the municipality of Vo, Italy. medRxiv Preprint posted online April 18, 2020. [doi: 10.1101/2020.04.17.20053157]

3. Flaxman S, Mishra S, Gandy A, Unwin HJT, Coupland H, Mellan TA, Imperial College London COVID-19 Response Team, et al. Estimating the number of infections and the impact of non-pharmaceutical interventions on COVID-19 in European countries: technical description update. arXiv Preprint posted online April 23, 2020.

4. Chu D, Akl E, Duda S, Solo K, Yaacoub S, Schünemann HJ, COVID-19 Systematic Urgent Review Group Effort (SURGE) study authors. Physical distancing, face masks, and eye protection to prevent person-to-person transmission of SARS-CoV-2 and COVID-19: a systematic review and meta-analysis. Lancet 2020 Jun 27;395(10242):1973-1987 [FREE Full text] [doi: 10.1016/S0140-6736(20)31142-9] [Medline: 32497510]

5. Chinazzi M, Davis JT, Ajelli M, Gioannini C, Litvinova M, Merler S, et al. The effect of travel restrictions on the spread of the 2019 novel coronavirus (COVID-19) outbreak. Science 2020 Apr 24;368(6489):395-400 [FREE Full text] [doi: 10.1126/science.aba9757] [Medline: $\underline{\text { 32144116] }}$

6. Fernandes N. Economic effects of coronavirus outbreak (COVID-19) on the world economy. SSRN J Preprint posted online March 23, 2020. [doi: 10.2139/ssrn.3557504]

7. Nussbaumer-Streit B, Mayr V, Dobrescu A, Chapman A, Persad E, Klerings I, et al. Quarantine alone or in combination with other public health measures to control COVID-19: a rapid review. Cochrane Database Syst Rev 2020 Apr 08;4:CD013574. [doi: 10.1002/14651858.CD013574] [Medline: 32267544]

8. Nicola M, Alsafi Z, Sohrabi C, Kerwan A, Al-Jabir A, Iosifidis C, et al. The socio-economic implications of the coronavirus pandemic (COVID-19): a review. Int J Surg 2020 Jun;78:185-193 [FREE Full text] [doi: 10.1016/j.ijsu.2020.04.018] [Medline: 32305533]

9. Jensen JB. Economic consequences of the COVID-19 pandemic. Copenhagen Economics. 2020 Mar. URL: https://www. copenhageneconomics.com/dyn/resources/Publication/publicationPDF/0/530/1585835646/ copenhagen-economics economic-consequences-covid-19.pdf

10. Holmes EA, O'Connor RC, Perry VH, Tracey I, Wessely S, Arseneault L, et al. Multidisciplinary research priorities for the COVID-19 pandemic: a call for action for mental health science. Lancet Psychiatry 2020 Jun;7(6):547-560. [doi: $10.1016 / \mathrm{s} 2215-0366(20) 30168-1]$

11. Coronavirus business impact: evolving perspective. McKinsey \& company. 2020. URL: https://www.mckinsey.com/ business-functions/risk/our-insights/covid-19-implications-for-business [accessed 2020-07-17] 
12. Lee V, Chiew C, Khong W. Interrupting transmission of COVID-19: lessons from containment efforts in Singapore. J Travel Med 2020 May 18;27(3) [FREE Full text] [doi: 10.1093/itm/taaa039] [Medline: $\underline{\text { 32167146] }}$

13. Ferguson NM, Cummings DAT, Fraser C, Cajka JC, Cooley PC, Burke DS. Strategies for mitigating an influenza pandemic. Nature 2006 Jul 27;442(7101):448-452 [FREE Full text] [doi: 10.1038/nature04795] [Medline: 16642006]

14. Osterholm MT, Hedberg CW. Epidemiologic principles. In: Mandell, Douglas, and Bennett's Principles and Practice of Infectious Diseases. Amsterdam: Elsevier; 2015:146-158.

15. Ye Q, Zhou J, Wu H. Using information technology to manage the COVID-19 pandemic: development of a technical framework based on practical experience in China. JMIR Med Inform 2020 Jun 08;8(6):e19515 [FREE Full text] [doi: 10.2196/19515] [Medline: 32479411$]$

16. Kamel Boulos MN, Geraghty EM. Geographical tracking and mapping of coronavirus disease COVID-19/severe acute respiratory syndrome coronavirus 2 (SARS-CoV-2) epidemic and associated events around the world: how 21st century GIS technologies are supporting the global fight against outbreaks and epidemics. Int J Health Geogr 2020 Mar 11;19(1):8 [FREE Full text] [doi: 10.1186/s12942-020-00202-8] [Medline: 32160889]

17. De Carli A, Franco M, Gassmann A, Killer C, Rodrigues B, Scheid E, et al. WeTrace -- a privacy-preserving mobile COVID-19 tracing approach and application. arXiv Preprint posted online April 19, 2020. [FREE Full text]

18. Cho H, Ippolito D, Yu YW. Contact tracing mobile apps for COVID-19: privacy considerations and related trade-offs. arXiv Preprint posted online March 25, 2020. [FREE Full text]

19. Xia Y, Lee G. How to return to normalcy: fast and comprehensive contact tracing of COVID-19 through proximity sensing using mobile devices. arXiv Preprint posted online April 5, 2020. [FREE Full text]

20. Yasaka TM, Lehrich BM, Sahyouni R. Peer-to-peer contact tracing: development of a privacy-preserving smartphone app. JMIR mHealth uHealth 2020 Apr 07;8(4):e18936 [FREE Full text] [doi: 10.2196/18936] [Medline: 32240973]

21. O'Neill PH, Ryan-Mosley T, Johnson B. A flood of coronavirus apps are tracking us. Now it's time to keep track of them. MIT Technology Review. 2020 May 07. URL: https://www.technologyreview.com/2020/05/07/1000961/ launching-mittr-covid-tracing-tracker/ [accessed 2020-07-17]

22. Altmann S, Milsom L, Zillessen H, Blasone R, Gerdon F, Bach R, et al. Acceptability of app-based contact tracing for COVID-19: cross-country survey evidence. medRxiv Preprint posted online May 8, 2020. [doi: $10.1101 / 2020.05 .05 .20091587$ ]

23. Ekong I, Chukwu E, Chukwu M. COVID-19 mobile positioning data contact tracing and patient privacy regulations: exploratory search of global response strategies and the use of digital tools in Nigeria. JMIR mHealth uHealth 2020 Apr 27;8(4):e19139 [FREE Full text] [doi: 10.2196/19139] [Medline: $\underline{32310817]}$

24. Abeler J, Bäcker M, Buermeyer U, Zillessen H. COVID-19 contact tracing and data protection can go together. JMIR mHealth uHealth 2020 Apr 20;8(4):e19359 [FREE Full text] [doi: 10.2196/19359] [Medline: 32294052]

25. Parker MJ, Fraser C, Abeler-Dörner L, Bonsall D. Ethics of instantaneous contact tracing using mobile phone apps in the control of the COVID-19 pandemic. J Med Ethics 2020 Jul;46(7):427-431 [FREE Full text] [doi:

10.1136/medethics-2020-106314] [Medline: 32366705]

26. Chen H, Yang B, Pei H, Liu J. Next generation technology for epidemic prevention and control: data-driven contact tracking. IEEE Access 2019;7:2633-2642. [doi: 10.1109/access.2018.2882915]

27. Segal E, Zhang F, Lin X, King G, Shalem O, Shilo S, et al. Building an international consortium for tracking coronavirus health status. Nat Med 2020 Aug;26(8):1161-1165. [doi: 10.1038/s41591-020-0929-x] [Medline: 32488218]

28. Pan-European Privacy-Preserving Proximity Tracing. 2020. URL: http://www.pepp-pt.org/

29. DP-3T / documents. GitHub. 2020. URL: https://github.com/DP-3T/documents

30. Whitepaper: slowing the spread of infectious diseases using crowdsourced data. Covid Watch. 2020. URL: https://www. covid-watch.org/covid watch whitepaper.pdf

31. Open-Source Project Corona-Warn-App. 2020. URL: https://www.coronawarn.app/en/ [accessed 2020-07-17]

32. Ding X, Clifton D, JI N, Lovell NH, Bonato P, Chen W, et al. Wearable sensing and telehealth technology with potential applications in the coronavirus pandemic. IEEE Rev Biomed Eng 2020 May 11. [doi: 10.1109/rbme.2020.2992838]

33. Ming DK, Sangkaew S, Chanh HQ, Nhat PT, Yacoub S, Georgiou P, et al. Continuous physiological monitoring using wearable technology to inform individual management of infectious diseases, public health and outbreak responses. Int $\mathrm{J}$ Infect Dis 2020 Jul;96:648-654 [FREE Full text] [doi: 10.1016/j.ijid.2020.05.086] [Medline: 32497806]

34. Tom-Aba D, Nguku PM, Arinze CC, Krause G. Assessing the concepts and designs of 58 mobile apps for the management of the 2014-2015 West Africa Ebola outbreak: systematic review. JMIR Public Health Surveill 2018 Oct 29;4(4):e68 [FREE Full text] [doi: 10.2196/publichealth.9015] [Medline: 30373727]

35. Ouedraogo B, Gaudart J, Dufour J. How does the cellular phone help in epidemiological surveillance? A review of the scientific literature. Inform Health Soc Care 2019 Jan;44(1):12-30. [doi: 10.1080/17538157.2017.1354000] [Medline: $\underline{28829691]}$

36. Munn Z, Peters MDJ, Stern C, Tufanaru C, McArthur A, Aromataris E. Systematic review or scoping review? Guidance for authors when choosing between a systematic or scoping review approach. BMC Med Res Methodol 2018 Nov 19;18(1):143 [FREE Full text] [doi: 10.1186/s12874-018-0611-x] [Medline: $\underline{\text { 30453902] }}$ 
37. Tricco A, Lillie E, Zarin W, O'Brien KK, Colquhoun H, Levac D, et al. PRISMA Extension for Scoping Reviews (PRISMA-ScR): checklist and explanation. Ann Intern Med 2018 Oct 02;169(7):467-473 [FREE Full text] [doi: 10.7326/M18-0850] [Medline: 30178033]

38. Abbas R, Michael K. COVID-19 contact trace app deployments: learnings From Australia and Singapore. IEEE Consumer Electron Mag 2020 Sep 1;9(5):65-70. [doi: 10.1109/mce.2020.3002490]

39. Ackley S, Pilewski S, Petrovic V, Worden L, Murray E, Porco T. Assessing the utility of a smart thermometer and mobile application as a surveillance tool for influenza and influenza-like illness. Health Informatics J 2020 Sep;26(3):2148-2158 [FREE Full text] [doi: 10.1177/1460458219897152] [Medline: $\underline{31969046]}$

40. Barrat A, Cattuto C, Tozzi A, Vanhems P, Voirin N. Measuring contact patterns with wearable sensors: methods, data characteristics and applications to data-driven simulations of infectious diseases. Clin Microbiol Infect 2014 Jan;20(1):10-16. [doi: 10.1111/1469-0691.12472] [Medline: 24267942]

41. Chan A, Drew D, Nguyen L, Joshi A, Ma W, Guo C, COPE Consortium. The COronavirus Pandemic Epidemiology (COPE) Consortium: a call to action. Cancer Epidemiol Biomarkers Prev 2020 Jul;29(7):1283-1289 [FREE Full text] [doi: 10.1158/1055-9965.EPI-20-0606] [Medline: $\underline{\text { 32371551] }}$

42. Farrahi K, Emonet R, Cebrian M. Epidemic contact tracing via communication traces. PLoS One 2014;9(5):e95133. [doi: 10.1371/journal.pone.0095133] [Medline: 24787614]

43. Ferretti L, Wymant C, Kendall M, Zhao L, Nurtay A, Abeler-Dörner L, et al. Quantifying SARS-CoV-2 transmission suggests epidemic control with digital contact tracing. Science 2020 May 08;368(6491) [FREE Full text] [doi: 10.1126/science.abb6936] [Medline: 32234805]

44. Jeong S, Kuk S, Kim H. A smartphone magnetometer-based diagnostic test for automatic contact tracing in infectious disease epidemics. IEEE Access 2019;7:20734-20747. [doi: 10.1109/access.2019.2895075]

45. Kim M, Yune S, Chang S, Jung Y, Sa S, Han H. The fever coach mobile app for participatory influenza surveillance in children: usability study. JMIR mHealth uHealth 2019 Oct 17;7(10):e14276 [FREE Full text] [doi: 10.2196/14276] [Medline: $\underline{31625946]}$

46. Kim M, Lee JY, Kim H. Warning and detection system for epidemic disease. 2016 Presented at: 2016 International Conference on Information and Communication Technology Convergence (ICTC); 2016; Jeju, South Korea p. $478-483$. [doi: 10.1109/ictc.2016.7763517]

47. Leal Neto O, Dimech GS, Libel M, de Souza WV, Cesse E, Smolinski M, et al. Saúde na Copa: the world's first application of participatory surveillance for a mass gathering at FIFA World Cup 2014, Brazil. JMIR Public Health Surveill 2017 May 04;3(2):e26 [FREE Full text] [doi: 10.2196/publichealth.7313] [Medline: 28473308]

48. Leal Neto O, Cruz O, Albuquerque J, Nacarato de Sousa M, Smolinski M, Pessoa Cesse EÂ, et al. Participatory surveillance based on crowdsourcing during the Rio 2016 Olympic Games using the Guardians of Health platform: descriptive study. JMIR Public Health Surveill 2020 Apr 07;6(2):e16119 [FREE Full text] [doi: 10.2196/16119] [Medline: 32254042]

49. Lwin MO, Vijaykumar S, Fernando ONN, Cheong SA, Rathnayake VS, Lim G, et al. A 21st century approach to tackling dengue: crowdsourced surveillance, predictive mapping and tailored communication. Acta Trop 2014 Feb;130:100-107. [doi: 10.1016/j.actatropica.2013.09.021] [Medline: 24161879]

50. Michael K, Abbas R. Behind COVID-19 contact trace apps: the Google-Apple partnership. IEEE Consumer Electron Mag 2020 Sep 1;9(5):71-76. [doi: 10.1109/mce.2020.3002492]

51. Miller A, Singh I, Koehler E, Polgreen P. A smartphone-driven thermometer application for real-time population- and individual-level influenza surveillance. Clin Infect Dis 2018 Jul 18;67(3):388-397. [doi: 10.1093/cid/ciy073] [Medline: $\underline{29432526]}$

52. Navin K, Mukesh Krishnan MB, Lavanya S, Shanthini A. A mobile health based smart hybrid epidemic surveillance system to support epidemic control programme in public health informatics. 2017 Presented at: 2017 International Conference on IoT and Application (ICIOT); 2017; Nagapattinam, India. [doi: 10.1109/iciota.2017.8073606]

53. Nguyen KA, Watkins C, Luo Z. Co-location epidemic tracking on London public transports using low power mobile magnetometer. 2017 Presented at: 2017 International Conference on Indoor Positioning and Indoor Navigation (IPIN); 2017; Sapporo, Japan. [doi: 10.1109/ipin.2017.8115963]

54. Olson D, Lamb M, Lopez MR, Colborn K, Paniagua-Avila A, Zacarias A, et al. Performance of a mobile phone app-based participatory syndromic surveillance system for acute febrile illness and acute gastroenteritis in rural Guatemala. J Med Internet Res 2017 Nov 09;19(11):e368 [FREE Full text] [doi: 10.2196/jmir.8041] [Medline: 29122738]

55. Okumura T. Tracing infectious agents with mobile location information: a simple and effective countermeasure against epidemic risks. 2019 Presented at: 2019 IEEE Global Humanitarian Technology Conference (GHTC); 2019; Seattle, WA. [doi: 10.1109/ghtc46095.2019.9033023]

56. Prieto JT, Jara JH, Alvis JP, Furlan LR, Murray CT, Garcia J, et al. Will participatory syndromic surveillance work in Latin America? Piloting a mobile approach to crowdsource influenza-like illness data in Guatemala. JMIR Public Health Surveill 2017 Nov 14;3(4):e87 [FREE Full text] [doi: 10.2196/publichealth.8610] [Medline: 29138128]

57. Rodriguez-Valero N, Luengo Oroz M, Cuadrado Sanchez D, Vladimirov A, Espriu M, Vera I, et al. Mobile based surveillance platform for detecting Zika virus among Spanish Delegates attending the Rio de Janeiro Olympic Games. PLoS One 2018;13(8):e0201943 [FREE Full text] [doi: 10.1371/journal.pone.0201943] [Medline: 30133492] 
58. Sugiura H, Ohkusa Y, Akahane M, Sugahara T, Okabe N, Imamura T. Construction of syndromic surveillance using a web-based daily questionnaire for health and its application at the G8 Hokkaido Toyako Summit meeting. Epidemiol Infect 2010 Oct;138(10):1493-1502. [doi: 10.1017/S095026880999149X] [Medline: 20067657]

59. Tripathy A, Mohapatra A, Mohanty S, Kougianos E, Joshi A, Das G. EasyBand: a wearable for safety-aware mobility during pandemic outbreak. IEEE Consumer Electron Mag 2020 Sep 1;9(5):57-61. [doi: 10.1109/mce.2020.2992034]

60. Vazquez-Prokopec G, Bisanzio D, Stoddard S, Paz-Soldan V, Morrison A, Elder J, et al. Using GPS technology to quantify human mobility, dynamic contacts and infectious disease dynamics in a resource-poor urban environment. PLoS One 2013;8(4):e58802 [FREE Full text] [doi: 10.1371/journal.pone.0058802] [Medline: 23577059]

61. Wang S, Ding S, Xiong L. A new system for surveillance and digital contact tracing for COVID-19: spatiotemporal reporting over network and GPS. JMIR mHealth uHealth 2020 Jun 10;8(6):e19457 [FREE Full text] [doi: 10.2196/19457] [Medline: 32499212]

62. Zhang Z, Wang H, Lin X, Fang H, Xuan D. Effective epidemic control and source tracing through mobile social sensing over WBANs. 2013 Presented at: 2013 Proceedings IEEE INFOCOM; 2013; Turin, Italy. [doi: 10.1109/infcom.2013.6566783]

63. Lewis DM. Frequently asked questions. CoEpi: Community Epidemiology in Action. 2020. URL: https://www.coepi.org/ $\underline{\text { faq/ }}$

64. Leith DJ, Farrell S. Coronavirus contact tracing: evaluating the potential of using bluetooth received signal strength for proximity detection. SIGCOMM Comput Commun Rev 2020 Oct 26;50(4):66-74. [doi: 10.1145/3431832.3431840]

65. Braz Sousa L, Fricker SR, Doherty SS, Webb CE, Baldock KL, Williams CR. Citizen science and smartphone e-entomology enables low-cost upscaling of mosquito surveillance. Sci Total Environ 2020 Feb 20;704:135349. [doi: 10.1016/j.scitotenv.2019.135349] [Medline: $\underline{31837870]}$

66. de Montjoye YA, Hidalgo C, Verleysen M, Blondel V. Unique in the crowd: the privacy bounds of human mobility. Sci Rep 2013;3:1376. [doi: 10.1038/srep01376] [Medline: 23524645]

67. Zang H, Bolot J. Anonymization of location data does not work: a large-scale measurement study. 2011 Presented at: Mobicom'11: The 17th Annual International Conference on Mobile Computing and Networking; 2011; Las Vegas, NV. [doi: $10.1145 / 2030613.2030630]$

68. Reichert L, Brack S, Scheuermann B. Privacy-preserving contact tracing of COVID-19 patients. IACR Cryptol ePrint Arch 2020.

69. Aktay A, Bavadekar S, Cossoul G, Davis J, Desfontaines D, Fabrikant A, et al. Google COVID-19 community mobility reports: anonymization process description. arXiv Preprint posted online April 8, 2020. [FREE Full text]

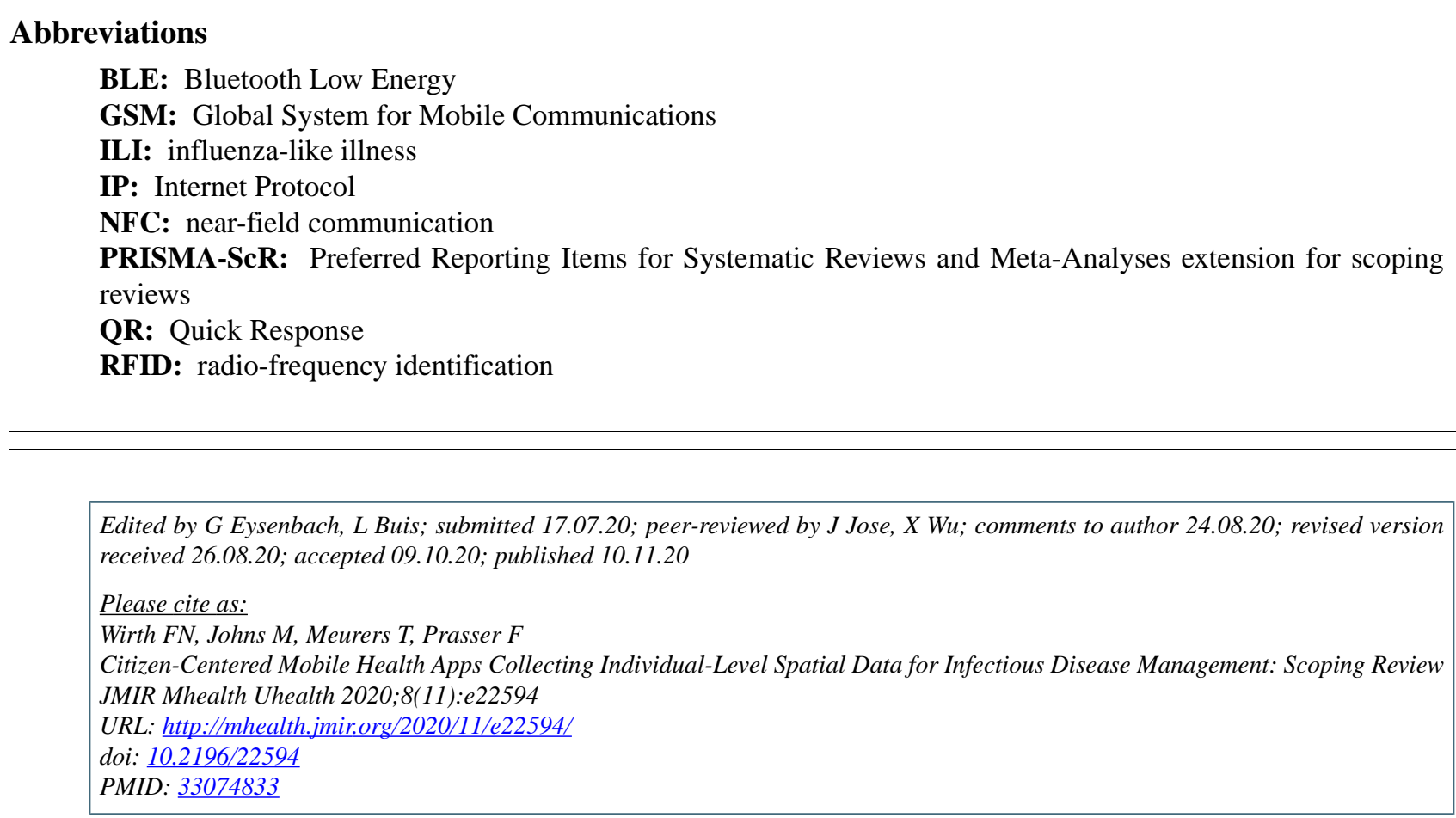

CFelix Nikolaus Wirth, Marco Johns, Thierry Meurers, Fabian Prasser. Originally published in JMIR mHealth and uHealth (http://mhealth.jmir.org), 10.11.2020. This is an open-access article distributed under the terms of the Creative Commons Attribution License (https://creativecommons.org/licenses/by/4.0/), which permits unrestricted use, distribution, and reproduction in any medium, provided the original work, first published in JMIR mHealth and uHealth, is properly cited. The complete bibliographic 
information, a link to the original publication on http://mhealth.jmir.org/, as well as this copyright and license information must be included. 